\title{
BMJ Open NHS Health Check programme: a rapid review update
}

\author{
L Tanner (1) , ${ }^{1}$ RPW Kenny, ${ }^{1}$ M Still, ${ }^{1}$ J Ling, ${ }^{2}$ F Pearson, ${ }^{1} \mathrm{~K}$ Thompson, ${ }^{3}$ \\ R Bhardwaj-Gosling ${ }^{1,2}$
}

To cite: Tanner L, Kenny RPW, Still M, et al. NHS Health Check programme: a rapid review update. BMJ Open 2022;12:e052832. doi:10.1136/ bmjopen-2021-052832

- Prepublication history and additional supplemental material for this paper are available online. To view these files, please visit the journal online (http://dx.doi.org/10.1136/ bmjopen-2021-052832).

Received 05 May 2021 Accepted 29 November 2021

Check for updates

(C) Author(s) (or their employer(s)) 2022. Re-use permitted under CC BY-NC. No commercial re-use. See rights and permissions. Published by BMJ.

${ }^{1}$ Population Health Sciences Institute, Newcastle University, Newcastle upon Tyne, UK ${ }^{2}$ Faculty of Health Sciences and Wellbeing, University of Sunderland, Sunderland, UK ${ }^{3}$ Public Health England, London, UK

Correspondence to

F Pearson;

Fiona.pearson2@newcastle. ac.uk

\section{ABSTRACT}

Objective To update a rapid review published in 2017, which evaluated the NHS Health Check programme. Methods An enlarged body of evidence was used to readdress six research objectives from a rapid review published in 2017, relating to the uptake, patient experiences and effectiveness of the NHS Health Check programme. Data sources included MEDLINE, PubMed, Embase, Health Management Information Consortium (HMIC), Cumulative Index of Nursing and Allied Health Literature (CINAHL), Global Health, PsycINF0, the Cochrane Library, NHS Evidence, Google Scholar, Google, ClinicalTrials.gov and the ISRCTN registry, Web of Science, Science Citation Index, The Cochrane Library, NHS Evidence, OpenGrey and hand searching article reference lists. These searches identified records from between January 1996 and December 2019. Screening, data extraction and quality appraisal using the Critical Appraisals Skills Programme checklists were performed in duplicate. Grading of Recommendations Assessment, Development and Evaluations was implemented. Data were synthesised narratively.

Results 697 studies were identified, and 29 new studies included in the review update. The number of published studies on the uptake, patient experiences and effectiveness of the NHS Health Check programme has increased by $43 \%$ since the rapid review published in 2017. However, findings from the original review remain largely unchanged. NHS Health Checks led to an overall increase in the detection of raised risk factors and morbidities including diabetes mellitus, hypertension, raised blood pressure, cholesterol and chronic kidney disease. Individuals most likely to attend the NHS Health Check programme included women, persons aged $\geq 60$ years and those from more socioeconomically advantaged backgrounds. Opportunistic invitations increased uptake among men, younger persons and those with a higher deprivation level.

Conclusions Although results are inconsistent between studies, the NHS Health Check programme is associated with increased detection of heightened cardiovascular disease risk factors and diagnoses. Uptake varied between population subgroups. Opportunistic invitations may increase uptake.

\section{INTRODUCTION}

The NHS Health Check (NHS-HC) programme is a cardiovascular disease (CVD) prevention programme introduced in 2009 aiming to assess all adults in England aged
Strengths and limitations of this study

This review summarises newly identified evidence, from January 1996 to December 2019, evaluating the NHS Health Check (NHS-HC) programme, building on an earlier rapid review published in 2017.

- The methods involved searches of published and grey literature sources, duplicate blinded screening, data extraction and quality appraisal and assessment of the quality of the overall body of evidence for each objective.

- Meta-analysis was not feasible due to the heterogeneous nature of the included studies.

- The results indicate that the NHS-HC programme increases the detection of individuals at risk of cardiovascular disease and that inequalities exist in NHS-HC attendance between population subgroups. Opportunistic invitations could increase uptake among these under-represented demographic groups.

- The overall body of evidence addressing the review objectives were 'very low' to 'moderate' quality therefore caution should be used when interpreting findings.

between 40 and 70 years old for CVD risk factors including obesity, physical inactivity, smoking and high alcohol consumption, high blood pressure and high cholesterol. Following assessment, using established tools, the level of individual risk is communicated to patients and evidence-based risk reduction interventions are implemented where appropriate. $^{12}$

An important aspect of the NHS-HC is the long-term goal of reducing inequalities in premature deaths from CVD, although the how was not explicitly stated. ${ }^{3}$ An observational study which used records from 9.5 million patients reported that NHS-HC attendees were more likely to be older and women, but were similar in terms of ethnicity and deprivation, compared with non-attendees. ${ }^{4}$ To address NHS-HC provider concerns ${ }^{5}$ regarding equity of access and to achieve the aim of reducing inequalities in 
premature CVD deaths, potential discrepancies in equity of access and outcomes must be identified and addressed.

Cost-effectiveness of the NHS-HC has been a focal point for discussion. Original modelling estimated the programme could prevent 1600 heart attacks and strokes, at least 650 premature deaths and over 4000 new cases of diabetes each year, with an estimated cost per quality adjusted life year (QALY) of approximately $£ 3000 .{ }^{6}$ Since then, it has been suggested that the programme is wasting large amounts of money ( $£ 450$ million) ${ }^{7}$ However, some evidence suggests the checks may be cost-effective, with small changes in body mass index (BMI) equating to a small but positive QALY gain of 0.05 per participant (cost-effectiveness ratio of $£ 900 /$ QALY). ${ }^{8}$ Additionally, such programmes could potentially be cost saving in the future if they correctly identify large numbers of people with CVD risk. ${ }^{9}$

Given these challenges it is important to consistently update and review available evidence to assess the impact of NHS-HC and the extent to which it is meeting the goal of addressing health inequalities. Additionally, a review of the NHS-HC programme was announced in the government's prevention green paper $^{10}$ and this evidence review was undertaken with the intention of informing that review and potential changes to policy. We therefore aimed to update a previously completed rapid synthesis of published research evidence on the NHS-HC programme, which incorporates evidence from studies published up to 9 November 2016. ${ }^{1}$ The main findings of this earlier review included that NHS-HCs are associated with small increases in disease detection. Higher attendance (number of attendees as a function of those who are eligible) was found among older people, women, the most deprived populations (which may reflect targeting) and non-smokers. Take-up (number of attendees as a function of those who are invited) of an NHS-HC varied between population subgroups, with older persons, women in younger age groups, men in older age groups and people from the least deprived areas were more likely to attend. People did not take up the offer of an NHS-HC due to factors including lack of awareness of the service, competing priorities and difficulty with getting a general practitioner (GP) appointment. Of those who attended NHS-HC, satisfaction levels were high. Methods which could increase uptake are invitation modifications and text message invitations or reminders. Health professionals expressed concerns regarding inequalities in uptake of the programme and the clinical and costeffectiveness of NHS-HC.

The rapid review reported here aimed to update the aforementioned review, using the same objectives (as stated below).

\section{OBJECTIVES}

Our aim was to update an earlier rapid review ${ }^{1}$ and summarise newly identified evidence addressing the following research objectives:
1. Who is and who is not having an NHS-HC?

2. What are the factors that increase take-up among the population and subgroups?

3. Why do people not take up an offer of an NHS-HC?

4. How is primary care managing people identified as being at risk of CVD or with abnormal risk factor results?

5. What are patients' experiences of having an NHS-HC?

6 . What is the effect of the NHS-HC on disease detection, changing behaviours, referrals to local risk management services, reductions in individual risk factor prevalence, reducing CVD risk and on statin and antihypertensive prescribing?

\section{METHODS}

A rapid review update reported according to the Preferred Reporting Items for Systematic Reviews and Meta-Analyses (PRISMA) guidelines. A checklist of PRISMA items is presented in the online supplemental file $\mathrm{S} 1 .^{11}$

\section{Patient and public involvement}

No patients involved.

\section{Literature searches}

The following databases were searched, from January 1996 to November 2016 in the earlier review ${ }^{1}$ and from January 2016 to December 2019 for this update: MEDLINE, PubMed, Embase, Health Management Information Consortium (HMIC), Cumulative Index of Nursing and Allied Health Literature (CINAHL), Global Health, PsycINFO, the Cochrane Library, NHS Evidence, Google Scholar, Google, ClinicalTrials.gov and the ISRCTN registry, Web of Science, Science Citation Index and OpenGrey. Hand searching of key article reference lists was also completed. The search strategy is available in the online supplemental file S2.

\section{Study selection}

Studies from the earlier review ${ }^{1}$ were included in the review update. The studies from updated searches were split into batches and each record was independently reviewed by two authors (either RPWK and LT or LT and FP) based on title, abstract and full text using prespecified inclusion and exclusion criteria (available in the online supplemental file S3) to identify those eligible for inclusion in the update. Conflicts were resolved through discussion, with adjudication by a third reviewer (either FP or RB-G depending on who had not previously reviewed the record) where necessary.

\section{Data extraction}

A random sample of $10 \%$ of the data extraction completed in the original review ${ }^{1}$ was checked by LT and found to be consistent with information reported in the primary studies. Data from newly identified studies were extracted onto prespecified, piloted, data proformas. Data from each quantitative study was extracted by a single reviewer (either RPWK or LT). Extracted data were then checked for accuracy by a different reviewer (either RPWK or 
LT). Any conflicts were resolved through discussion or via adjudication by a third reviewer (FP) when necessary. Pertinent qualitative data including direct participant quotes, researcher interpretations and concepts were extracted in duplicate (by MS and FP) with discrepancies discussed and resolved. Data were coded against the themes previously identified. ${ }^{1}$ Emergent themes not previously identified were discussed and coded (by MS and FP). Duplicate extraction was completed for each qualitative paper by two reviewers from differing standpoints so as not to subconsciously affect the data being extracted and synthesised.

\section{Quality appraisal}

The quality of newly identified studies was assessed by a single reviewer then verified by a second. Any discrepancies were resolved through discussion and, where required, adjudicated by a third reviewer. Qualitative studies were assessed by MS or FP using The Critical Appraisal Skills Programme (CASP) checklist for qualitative research. ${ }^{12}$ Quantitative studies were assessed by RPWK or LT using a tool that was developed using CASP tools ${ }^{12}$ and implemented by the previous review authors ${ }^{1}$ to accommodate the range of study designs included.

\section{Data synthesis}

Synthesis of new quantitative and qualitative data were completed as an extension to that undertaken in the original review. Numerical data were combined using a structured, narrative synthesis. Meta-analysis was not methodologically appropriate due to high heterogeneity and a low number of high-quality studies reporting on each objective in a consistent manner. For the qualitative data, a three-stage thematic synthesis approach ${ }^{13}$ was planned in which newly identified studies could add to and potentially revise the original findings. This approach involves 'line-by-line' coding of the findings according to the content and meaning; developing 'descriptive themes' by grouping codes according to similarities and differences; generating 'analytical themes' based on the reviewer's interpretation of the data in relation to the research question. ${ }^{13}$

\section{Assessment of the certainty of the evidence}

Grading of Recommendations Assessment, Development and Evaluations (GRADE), ${ }^{14}$ GRADE-Confidence in the Evidence from Reviews of Qualitative Research (GRADECERQual) ${ }^{15}$ and a method for assessing certainty of evidence in mixed methods reviews ${ }^{16}$ were used to assess the certainty and confidence in quantitative, qualitative and mixed methods evidence, respectively, contributing to each objective and subobjective as appropriate.

\section{RESULTS}

The PRISMA flow diagram of included and excluded studies is shown in figure 1.
Twenty-nine newly identified studies were eligible for inclusion. The numbers of newly identified studies mapping to each research objective are as follows: objective $1(n=6)$, objective $2(n=9)$, objective $3(n=0)$, objective $4(n=4)$, objective $5(n=2)$ and objective $6(n=13)$. Quality appraisal scores for each study are shown in online supplemental file S4. GRADE assessments are shown in online supplemental file S5. The overall certainty of evidence ranged from 'very low' to 'moderate'. Results are also synthesised below in relation to each objective and subobjective.

\section{Objective 1: differences in demographics of those attending} and not attending an NHS-HC

NHS digital and Public Health England (PHE) published attendance data from 2012 to 2018. ${ }^{17}$ The national average attendance was $44.2 \%$, with variation across regions $($ range $=41.3 \%-49.2 \%)$. The variation was greater at a local authority level where 2017-2018 attendance varied from $19.5 \%$ to $75.8 \%$. The original review identified 24 studies for this objective. This update identified six new studies.

Generally, more older adults (eg, >60 years old) attended than younger adults. ${ }^{18-20}$ Evidence suggested men are less likely to attend than women, ${ }^{17-19} 2122$ as statistically evidenced in $^{21}$ (adjusted OR (AOR): 0.75, 95\% CI: 0.67 to 0.84 ) and $^{19}$ (AOR: $0.73,95 \%$ CI: 0.67 to 0.8 ). Another study ${ }^{20}$ however, provide some evidence that men may be more likely to attend than women when the NHS-HCs were conducted opportunistically, where health checks are offered to patients during face-to-face medical consultations for other reasons.

Attendance data regarding ethnic groups is inconclusive. The NHS Digital data ${ }^{17}$ shows that over the time period of 2012-2018, those of an Asian or black background had greater numbers of attendance than not attendance. While those of a white British background had a greater number of non-attendees compared with attendees. However, this varied greatly by year with no single ethnic group consistently attending more often than not attending. ${ }^{17}{ }^{18}$ The authors of one study, ${ }^{18}$ however, claim that white British had greater attendance at a national level but given that white British make up most of the eligible population this finding could be misleading. Attendance by ethnicity probably varies depending on location. For example, community data from Leicester showed that people from black and minority ethnic groups were more likely to attend than white people. ${ }^{20}$ In terms of socioeconomic status, there is some evidence those from a higher level of deprivation (identified by Index of Multiple Deprivation (IMD)) are less likely to attend an NHS-HC. ${ }^{19}{ }^{20}$ However, opportunistic NHS-HCs show an increase in attendance from those of a higher deprivation level. ${ }^{22}$

There is evidence to suggest lower levels of NHS-HC attendance among smokers. ${ }^{2021}$ One study ${ }^{20}$ also reported the effect of religion on attendance, suggesting higher attendance of non-Christians than Christians. Those with 


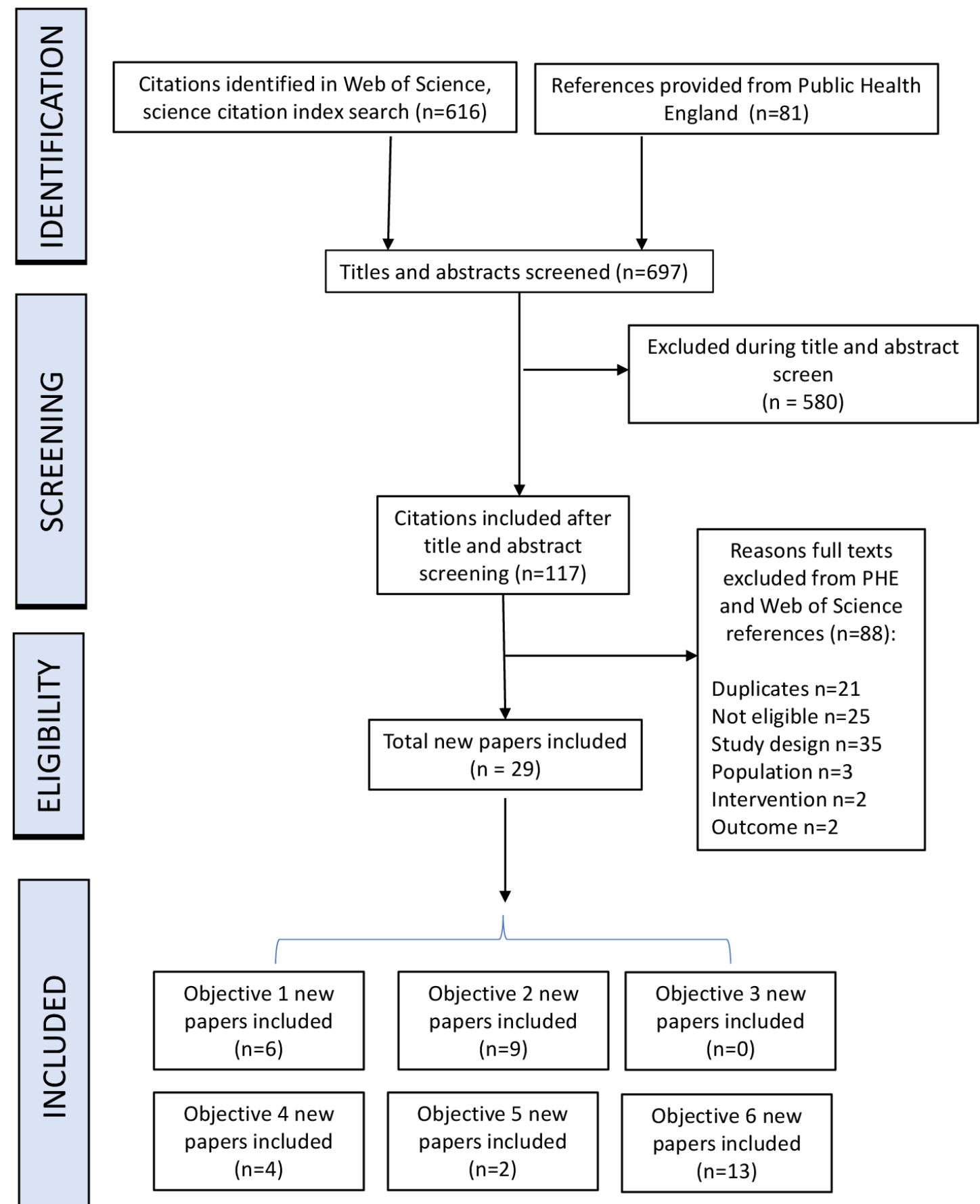

Figure 1 Preferred Reporting Items for Systematic Reviews and Meta-Analyses flow chart depicting the flow of included and excluded studies.

no religious background were less likely to attend overall. This finding was from a single small community-based study and it is, therefore, difficult to make any inferences about the wider population.

The GRADE certainty in evidence rating for Objective 1 was 'low' due to the observational nature of study designs that contributed evidence.

\section{Objective 2: what factors increase take-up among population} and subgroups?

Uptake has maintained a range of $45 \%-50 \%$, with recent national data from PHE reporting an uptake of $45.9 \%$ for $2018 / 2019 .^{23}$ There are, however, variations by region and constituency. For example, in the North East uptake varied between $25 \%$ and $61 \%$.

Objective 2.1: socio-demographic determinants of uptake

There were 11 quantitative studies included in the original review. We identify one new quantitative study conducted in two London boroughs (18 GP practices) reporting socio-demographic differences in uptake. ${ }^{24} \mathrm{~A}$ randomised control trial (RCT) assessing uptake via standard invitation letter or a question behaviour effect (QBE) questionnaire (with/without financial incentive) followed by the 
invitation letter. Uptake across the three trial arms was $15.3 \%$. This is significantly lower than previously reported $\left(27 \% \mathrm{in}^{25} ; 34.1 \% \mathrm{in}^{26}\right.$ and $\left.44.8 \% \mathrm{in}^{27}\right)$. One study ${ }^{24}$ also found men and younger people less likely to attend an NHS-HC. Those with a non-white ethnic background were more likely to attend, however, this study area includes a large proportion of individuals from a non-white ethnic background and results may not be reflective of the wider population. Contradictory to Objective 1 findings, those from the second least deprived quintile were more likely to attend than those from the most deprived.

\section{Objective 2.2: invitation methods}

Six new studies, adding to seven previously identified, assess the effects of different invitation methods, compared with the standard invitation letter, on uptake. ${ }^{24-32}$ Use of the QBE questionnaire alone or with a financial incentive $(£ 5)$ did increase uptake when it was returned. There were, however, no statistically significant changes in risk difference between the two invitation types $(1.52 \%, 95 \%$ CI: $-0.03 \%$ to $3.07 \%, p=0.054)$. This is lower than previous research estimating a $3 \%-4 \%$ change. ${ }^{33}$ One study compared the use of modified letters and telephone invitations. ${ }^{30}$ While a different study compared a letter with yes/no SMS (short messaging service) pre and post invitation. ${ }^{32}$ Another study implemented new shorter leaflet styles (two vs four pages) but there were no statistically meaningful changes in uptake. ${ }^{31}$ Use of SMS reminders and time limited letters did, increase uptake ${ }^{31}$; confirming the positive results previously reported in a similar study. ${ }^{34}$ Telephone invitations also improved uptake compared with the standard letter invitation and a personalised CVD risk. ${ }^{30} \mathrm{~A}$ cost analysis suggests that for every 1000 patients invited by telephone (compared with standard letters) an additional 180 NHS-HCs could be expected, with an extra cost of $£ 0.24$ / patient. Telephone invitations are also strongly preferred by primary care and outreach workers. ${ }^{35}$ Finally, the use of opportunistic invitations compared with the standard invitation letter improved uptake of those identified at greater CVD risk (ie, risk score 10\%). ${ }^{29}$ Using opportunistic invitations also lead to an increase in younger patients attending. ${ }^{22}$

\section{Objective 2.3: setting}

This update identified two quantitative studies which assessed the impact of setting on uptake rates; none were identified in the earlier review. These studies compared a GP setting to an outreach service ${ }^{36}$ or community pharmacy. ${ }^{37}$ One of the studies targeted hard-to-reach groups using opportunistic methods. While GP attendance was three times more than the outreach services, people of a South Asian ethnicity and higher IMD were more likely to attend the outreach services. ${ }^{36}$ Men, however, were more likely to attend a GP than an outreach or community pharmacy service. ${ }^{36} 37$ The other study found minimal differences in uptake of NHS-HCs after invitation by letter. ${ }^{37}$ Opportunistic methods may provide greater uptake in some harder-to-reach patients.
The GRADE certainty in evidence ratings for Objectives 2.1-3 ranged from 'low' due to the observational nature of study designs to 'very low' due to high risk of bias ratings.

Objective 3: why do people not take up an offer of an NHS-HC? No new studies identified addressed this objective.

\section{Objective 4: how primary care is managing people identified} as being at risk of CVD or with abnormal risk factor results The only study across both reviews to focus on risk management was. ${ }^{38}$ They assessed CVD risk factors in England over a 6 -year follow-up period. An interrupted time series analysis revealed mean BMI following a health check was $0.3 \mathrm{~kg} / \mathrm{m}^{2}$ (95\% CI: 2 to $\left.0.39 \mathrm{~kg} / \mathrm{m}^{2}\right)$ lower, while control patients' (no health check) BMI increased $\left(0.08 \mathrm{~kg} / \mathrm{m}^{2}\right.$, 95\% CI: 0.07 to $0.09 \mathrm{~kg} / \mathrm{m}^{2}$ per year). ${ }^{38}$ Additionally, after the 6-year period, patients who had a health check were less likely to be smokers (AOR: $0.9,95 \%$ CI: 0.87 to 0.94 ). NHS-HC attendees also had lower systolic and diastolic blood pressure, and lower total cholesterol. ${ }^{38}$ High density lipoprotein was, however, slightly higher after 6 years $(0.01,95 \%$ CI: 0.002 to 0.02$)$. This single large study provides evidence that NHS-HCs can increase provision of risk management advice and interventions.

Fifteen qualitative studies were identified by the previous review, a further three are presented here. Three qualitative studies ${ }^{35} 3940$ investigated the views of those responsible for delivery of NHS-HCs. Healthcare professionals interviewed by ${ }^{39}$ suggested that an NHS-HC was unlikely to be successful because people already knew the positive health behaviours they needed to engage with, but chose to ignore public health messaging. In a later study ${ }^{40}$ it was found that GPs seemed more negative towards delivery of NHS-HCs than other staff. NHSHCs were seen as time consuming or unclear in terms of outcome. Several GPs felt that it would be more efficient if healthcare assistants (HCAs) conducted the NHS-HC as the HCAs role is more focused on health promotion activities so they are more likely to have the opportunity and skills to elicit more personal information from patients. In contrast, HCAs were unsure if they had the right skills to undertake NHS-HCs, and indeed, whether this should be part of their role. One study found health professionals thought it was beneficial to have someone from a similar ethnic background invite a patient for an NHS-HC, as they understood how certain elements of the NHS-HC would relate to specific communities. ${ }^{35}$ They also identified that employing outreach workers freed up GP and practice staff time to focus on other tasks. However, as outreach staff worked across multiple practices in the district, some practice managers were negative about the system as it meant they did not operationally manage them.

The certainty in evidence rating for Objective 4 was 'moderate'. Lack of objectivity was the main area of concern across studies addressing this objective. 


\section{Objective 5: patient views on NHS-HCs}

One study found patients felt a sense of obligation to attend and be 'a willing patient', but family history affected how likely they were to make a change. ${ }^{41}$ Some pointed to longevity in their family as a reason to avoid changing their health behaviours, others felt that as family members had high risk of CVD disease, it was inevitable they too would experience high risk, regardless of any behaviour change. In two studies by the same author $^{39} 40$ patients could not recall a specific risk score but did remember discussions around their current state of health. People felt more able to make changes when their family and friends supported and facilitated them to do so. Individuals valued being able to use their results from their NHS-HC to converse with their support networks identifying and introducing changes to their behaviours. While one patient found the form filling and nature of the questioning to be off-putting, ${ }^{41}$ the majority felt the experience of having a health check was positive.

The certainty in evidence rating for Objectives 5 was 'low' due to the subjective nature of participant data, to 'moderate'.

\section{Objective 6: effects of the NHS-HC programme on health outcomes}

Studies mapped to Objective 6 assessed the effects of the NHS-HC on one of the following predefined health outcomes: disease detection, changing behaviours, referrals to local risk management services, reductions in individual risk factor prevalence, reducing CVD risk and statin and antihypertensive prescribing.

\section{Objective 6.1: disease detection}

Seventeen studies reported data on disease detection, five of these were newly identified. One of the newly identified studies used data from 455 GP practices across England. ${ }^{42}$ Incidence rates of detected non-diabetic hyperglycaemia and type 2 diabetes were significantly higher among individuals registered at GP surgeries with high NHS-HC coverage, compared with low coverage surgeries. Rates of non-diabetic hyperglycaemia were reported to be $19 \%$ higher in the high coverage compared with the low coverage group (HR 1.19, 95\% CI: 1.01 to 1.41 ) and rates of type 2 diabetes were $10 \%$ higher (HR 1.11, 95\% CI: 1.03 to 1.19$).^{42}$

Four studies used samples from smaller areas of England. One of the studies reported that individuals who received opportunistic NHS-HCs offered during patient encounters for other reasons, were significantly more likely to have a higher 10-year risk of CVD (CVD risk score $\geq 10 \%$, assessed using the Joint British Societies' 'JBS3' risk calculator) compared with individuals who chose to attend following an invitation. ${ }^{29}$ Two studies reported that NHS-HC attendance compared with nonattendance was associated with significant increase in detection or diagnosis of the following conditions: CVD risk $>10 \%{ }^{43}$; diabetes and hypertension, ${ }^{43} 44$ total cholesterol $^{43}$ and chronic kidney disease (CKD). ${ }^{44} \mathrm{~A}$ different study compared disease detection rates between NHS-HC attendees from different socioeconomic groups and reported a significant increase in the detection of CVD risk $>20 \%$ among individuals from the most deprived IMD decile. $^{21}$

\section{Objective 6.2: health-related behaviours}

Five studies (one newly identified) reported data on health-related behaviours. The newly identified study used national (England) data from the Clinical Practice Research Datalink data set. NHS-HC participants were less likely to be smokers compared with a control group after 6 years' follow-up (health check 17\% vs controls 25\%; OR 0.90, 95\% CI: 0.87 to $0.94, \mathrm{p}<0.001$ ) however, a greater reduction in smoking prevalence was reported for the control group. ${ }^{38}$

\section{Objective 6.3: risk management referrals}

Ten studies (four newly identified) reported data quantifying the proportion of NHS-HC attendees who were referred to lifestyle services. Two of the new studies used data from across England, ${ }^{40}$ one study involved a sample of 151 general practices in Hampshire ${ }^{43}$ and the other from 38 GP practices in Bristol. ${ }^{19}$

The proportions of NHS-HC attendees who were offered risk management advice or referrals varied between studies and in relation to the risk factor addressed, from $1.8 \%$ to $90 \%$ for smoking cessation interventions, <1\%-73\% for weight management interventions among patients with a BMI of $\geq 30$ and between $0.01 \%$, and $33.9 \%$ for interventions to reduce alcohol consumption among patients who consumed $\geq 14$ units per week. This is likely reflective of geographical variations in referrals between areas.

\section{Objective 6.4: CVD risk}

Five studies (one newly identified) assessed the change in CVD risk factor values following the NHS-HC. The newly identified study used national data from across England. Adjusted mean differences in 10-year CVD risk scores between intervention recipients and non-recipients at 6 years post-NHS-HC, were as follows: BMI $\left(\mathrm{Kg} / \mathrm{m}^{2}\right)-0.30$ (95\% CI: -0.39 to $-0.20, \mathrm{p}<0.001)$; systolic blood pressure (mean, $\mathrm{mm} \mathrm{Hg}$ ) -1.43 (95\% CI: -1.70 to -1.16 , p<0.001); diastolic blood pressure (mean, $\mathrm{mm} \mathrm{Hg})-0.93(95 \%$ CI: -1.11 to $-0.75, \mathrm{p}<0.001$ ) total cholesterol (mean, $\mathrm{mmol} / \mathrm{L})-0.05$ (95\% CI: -0.07 to $-0.03, \mathrm{p}<0.001)$, high density lipoprotein cholesterol (mean, mmol/L) 0.01 (95\% CI: 0.002 to $0.02, \mathrm{p}>0.05) .^{38}$

\section{Objective 6.5: prescribing of statins and antihypertensives}

Sixteen studies (four newly identified) reported data on prescribing after the implementation of NHS-HC. One of the newly identified studies which used national data from across England reported that NHS-HC participants were more likely to receive statins (HR 1.24, 95\% CI: 1.21 to $1.27, \mathrm{p}<0.001)$ and were less likely to receive antihypertensive drugs (HR $0.86,95 \%$ CI: 0.85 to 0.88 , $\mathrm{p}<0.001)$ compared with non-attendees. ${ }^{38}$ One study found that new statin prescriptions were higher for 
NHS-HC attendees compared with non-attendees. ${ }^{44}$ The proportions of new statin prescriptions administered to NHS-HC attendees versus non-attendees were $11.5 \%$ and $8.2 \%$, respectively. These data were from 143 general practices in three clinical commissioning groups in east London (England, UK). A different study also reported that NHS-HCs led to increased use of statins (OR 1.54, 95\% CI: 1.39 to 1.71$)$ in addition to antihypertensives (OR 1.15, 95\% CI: 1.06 to 1.24) using data from 151 GP practices in Hampshire. ${ }^{43}$ Another study compared prescribing rates between population subgroups (men/ women and age group) among NHS-HC attendees using data from GP practices in Bristol. ${ }^{19}$ The results indicated that women were more likely than men to be prescribed a cardiovascular drug, (OR 1.18, 95\% CI: 1.03 to 1.35 ) as were patients aged $\geq 70$ years compared with aged $\leq 70$ years (OR 1.64, 95\% CI: 1.14 to 2.35 ). In the same study, individuals classified as being at high risk of CVD were most likely to be prescribed CVD medication (OR 6.16, 95\% CI: 4.51 to 8.40 ). There was no evidence of any association between the prescribing of CVD medication and socioeconomic status or ethnicity.

\section{Objective 6.6: economic modelling studies}

Six studies (three newly identified) assessed the costeffectiveness of the NHS-HC programme based on different implementation approaches. Two of the new studies, which are related, assessed implementation and redesign scenarios using demographic data from Liverpool's population, exposure to risk factors and CVD epidemiology to assess health benefits, equity and costeffectiveness. ${ }^{46} 47$ The third study assessed whether the impact of the checks on BMI was sufficient to justify its costs. ${ }^{48}$ The two related studies reported that the equitability and cost-effectiveness of the NHS-HC programme would be increased through the addition of policies targeting dietary consumption and through combining current provision with targeting of the intervention in deprived areas. ${ }^{464}$ The third study reported that even modest changes in BMI from the NHS-HC programme are associated with significant cost-saving benefits making the programme cost-effective. ${ }^{48}$

The GRADE certainty in evidence ratings for Objectives 6.1-5 ranged from 'very low' due to risk of bias, indirectness, imprecision and inconsistency, to 'moderate'.

\section{DISCUSSION}

The goal of the NHS-HC programme is to identify and reduce CVD risk in those aged between 40 and 74 years. This rapid review aimed to update existing evidence on a previously completed review.

\section{Principal findings}

The proportion of published studies has increased by $43 \%$ since the earlier review. ${ }^{1}$ However, the majority of the key findings from the original review remain unchanged in this review update. The overall results from the earlier review and the review update are summarised as follows for each objective along with the findings from a body of relevant evidence identified prior to the publication of this review:

\section{Objective 1: who is and who is not having an NHS-HC?}

There is higher NHS-HC attendance among women and people aged 60 years and over. The association between female gender and NHS-HC attendance was confirmed by a newly identified study. ${ }^{49}$ The evidence synthesised in this review indicated that smokers and those from high levels of deprivation are least likely to take up an invitation to attend an NHS-HC, although a more recent study on over 9.5 million people reported no significant evidence of inequity of attendance by deprivation level. ${ }^{4}$ There is mixed evidence regarding the association between ethnicity and NHS-HC attendance. Newly located studies report higher attendance among South Asian ethnic groups $^{49}$ and people with serious mental illnesses. ${ }^{50}$

Objective 2: what are the factors that increase take-up among the population and subgroups?

Opportunistic invitations, telephone invitations and text message reminders increased uptake compared with the standard invitation letters. Additionally, delivery setting influenced uptake in population subgroups, with people of a South Asian ethnicity and higher IMD more likely to attend the outreach services. ${ }^{35}$ An RCT published in 2021 found that automated prompts to clinical staff to invite patients to NHS-HCs, delivered via computer systems in general practice, improved uptake, especially for men and younger patients. ${ }^{51}$

\section{Objective 3: why do people not take up an offer of an NHS-HC?}

The earlier review ${ }^{1}$ reported that lack of awareness or knowledge, competing priorities, misunderstanding the purpose, an aversion to preventive medicine, difficulty getting an appointment with a GP and concerns about privacy and confidentiality reduced NHS-HC attendance among the general population. A newly identified study, published in 2020, identified barriers to NHS-HC uptake among prisoners, which included poor accessibility to the healthcare department, stigma of visiting healthcare and fear surrounding the NHS-HC. ${ }^{52}$

Objective 4: how is primary care managing people identified as being at risk of CVD or with abnormal risk factor results?

We found variations in risk management referrals across the reviewed studies, possibly reflecting geographical variations. A newly retrieved study reported that overall fidelity of delivery of NHS-HCs in general practice was high, however, important elements of the NHS-HC, including assessments in relation to ethnicity and family history of disease, in addition to the Alcohol Use Disorders Identification Test and dementia risk management, were being regularly omitted. ${ }^{53}$ Another new study found that practitioners often demonstrated limited understanding and confidence in explaining the 10-year risk score to patients, whereas confidence in the JBS3 lifetime 
CVD risk calculator, with its visual information summaries, was higher. ${ }^{54}$

\section{Objective 5: patient views on the NHS-HC programme}

Overall patient satisfaction levels with the programme were high, however the risk score was less helpful to patients than discussion about their health with the clinician during the NHS-HC. Although more recent research suggests that visual representations of CVD risk were more easily understood than a percentage risk score. ${ }^{55}$ Behaviour change may be influenced by perceived risk based on family history and social support. A newly identified study reported that participants did not like the form-filling aspect of the NHS-HC. ${ }^{56}$

\section{Objective 6: what is the effect of the NHS-HC on disease} detection...?

Overall, the NHS-HC programme is associated with increased detection of CVD risk factors and diagnoses, increased prescribing of cardiovascular medications and with a general reduction in CVD risk factors. The results from two newly identified studies confirmed these findings. ${ }^{49} 57$ The economic evidence indicated that the cost-effectiveness of the NHS-HC programme varies; population-wide interventions were more costeffective than individual level interventions and interventions targeted at deprived areas were more cost-effective compared with non-targeted interventions. A study published in 2020 found that people with serious mental illnesses were more likely to: attend an NHS-HC; have higher rates of CKD and type 2 diabetes; and have received treatment with statins and antihypertensive medication, compared with people without these conditions. ${ }^{50}$

\section{Strengths and weaknesses of the study}

The methods used to review the evidence available on the NHS-HC programme involved searches of published and grey literature sources, duplicate blinded screening, data extraction and quality appraisal and assessment of the quality of the overall body of evidence for each objective. Methods used to synthesise the new data with the existing body of evidence were appropriate given the quantity and types of new studies identified. Review limitations included that it was not possible to perform meta-analysis due to the heterogeneous nature of the included studies. The use of 'vote counting' methods potentially compromises the precision of the results. ${ }^{58}$ Also, the searches undertaken for this review update were completed in December 2019, 2 years prior to publication of this manuscript. The evidence presented therefore, does not include more recent publications.

\section{Strengths and weaknesses of the available evidence}

General consistency of findings across studies in relation to each review objective supports causal inferences regarding the direction of effect of the NHS-HC programme on the health-related outcomes assessed. The overall quality of evidence varied between objectives and ranged from 'very low' to 'moderate', reflecting issues including that most studies were observational with confounding and poor internal validity (assessed using risk of bias). Furthermore, inconsistent data collection and reporting across many of the studies reduces precision of estimated effect of the NHS-HC programme on health-related outcomes.

\section{Implications for policy and practice}

The results from this review could inform changes to the methods used to invite eligible individuals to attend an NHS-HC, for example, by modifying the invitation method (eg, telephone invitations and sending text message reminders). Opportunistic recruitment could be used to selectively target specific groups who are at greater risk, as well as those who are less likely to engage with the NHS-HC programme.

\section{Unanswered questions and future research}

There is a need to understand more fully the effect of the programme on lifestyle behaviours including further research to explore the impact of attending an NHS-HC on physical activity, diet and alcohol consumption. The identified barriers to the uptake of an NHS-HC need to be explored in more depth as they could inform improvement of recruitment to the programme. In particular, future research should examine the potential of NHS-HC to widen inequalities given the demographics of participants identified in our review. A review of interventions for CVD (eg, physical activity or diet change), outside of the NHS-HC programme could help inform further development of the programme.

\section{CONCLUSIONS}

The NHS-HC programme increases the detection of individuals at risk of CVD. The overall body of evidence addressing the review objectives were 'very low' to 'moderate' quality therefore caution should be used when interpreting findings, which appear to show that inequalities exist in NHS-HC attendance between population subgroups. There are also geographical variations rates of referral to lifestyle services following NHS-HC. Targeting NHS-HC towards high-risk communities (eg, deprived communities) may increase the cost-effectiveness of the programme. Uptake may be increased through opportunistic invitations in addition to addressing misconceptions regarding the purpose, importance and confidential nature of the programme. Discussion between NHS-HC attendees regarding their health and their GP may be more helpful than receiving a risk score, which may not be understood or remembered by the patient. Family history of disease and social support could determine the impact of the intervention on behaviour change.

Acknowledgements Eleanor Wilkinson was involved in conceptualisation and design of the review.

Contributors FP acts as gaurantor. FP, KT and RB-G conceptualised and designed the review. Literature searches were designed and implemented by Public Health England's Information Specialist Team and FP. FP, LT, RB-G and RPWK reviewed 
titles, abstracts and full-text papers for eligibility. FP, LT, MS, RB-G and RPWK completed data extraction and quality appraisal. The manuscript was prepared by FP, LT, MS and RPWK. JL, KT and RB-G provided critical revision.

Funding The funding to conduct the project was awarded by Public Health England [grant number 191209]. In addition, JL received funding for open-access publication costs from Fuse, the Centre for Translational Research in Public Health ( www.fuse.ac.uk). The views expressed in this paper do not necessarily represent those of the funders or UKRI and the funders had no role in study design, data collection and analysis, decision to publish, or preparation of the manuscript.

Competing interests The research funding for this project was won by academics from Sunderland and Newcastle Universities in an open national competition from Public Health England (PHE). KT is Head of the Cardiovascular Disease Prevention Programme at PHE.

Patient consent for publication Not applicable.

Ethics approval This study does not involve human participants.

Provenance and peer review Not commissioned; externally peer reviewed.

Data availability statement All data relevant to the study are included in the article or uploaded as supplementary information. Not applicable.

Supplemental material This content has been supplied by the author(s). It has not been vetted by BMJ Publishing Group Limited (BMJ) and may not have been peer-reviewed. Any opinions or recommendations discussed are solely those of the author(s) and are not endorsed by BMJ. BMJ disclaims all liability and responsibility arising from any reliance placed on the content. Where the content includes any translated material, BMJ does not warrant the accuracy and reliability of the translations (including but not limited to local regulations, clinical guidelines, terminology, drug names and drug dosages), and is not responsible for any error and/or omissions arising from translation and adaptation or otherwise.

Open access This is an open access article distributed in accordance with the Creative Commons Attribution Non Commercial (CC BY-NC 4.0) license, which permits others to distribute, remix, adapt, build upon this work non-commercially, and license their derivative works on different terms, provided the original work is properly cited, appropriate credit is given, any changes made indicated, and the use is non-commercial. See: http://creativecommons.org/licenses/by-nc/4.0/.

\section{ORCID iD}

L Tanner http://orcid.org/0000-0003-2340-8677

\section{REFERENCES}

1 Usher-Smith J, Mant J, Martin A. NHS health check programme rapid evidence synthesis. University of Cambridge, 2017.

2 National Health Service. NHS health checks, 2021. Available: https:// www.england.nhs.uk/ltphimenu/cvd/nhs-health-checks/

3 Honey S, Bryant LD, Murray J, et al. Differences in the perceived role of the healthcare provider in delivering vascular health checks: a Q methodology study. BMC Fam Pract 2013;14:1-14. doi:10.1186/1471-2296-14-172

4 Patel R, Barnard S, Thompson K, et al. Evaluation of the uptake and delivery of the NHS health check programme in England, using primary care data from 9.5 million people: a cross-sectional study. BMJ Open 2020;10:e042963.

5 Mills K, Harte E, Martin A, et al. Views of commissioners, managers and healthcare professionals on the NHS health check programme: a systematic review. BMJ Open 2017;7:e018606.

6 Expert Scientific and Clinical Advisory Panel. Emerging evidence on the NHS health check: findings and recommendations, 2008.

7 Wise J. NHS health check programme wastes£ 450m a year, report says. British Medical Journal Publishing Group, 2015.

8 Hinde S, Bojke L, Richardson G, et al. The cost-effectiveness of population health checks: have the NHS health checks been unfairly maligned? Z Gesundh Wiss 2017;25:425-31. doi:10.1007/s10389017-0801-8

9 Thomas C, Brennan A, Goka E, et al. What are the cost-savings and health benefits of improving detection and management for six high cardiovascular risk conditions in England? an economic evaluation. BMJ Open 2020;10:e037486.

10 Department of Health and Social Care. Advancing our health: prevention in the 2020s - consultation document, 2019.

11 Moher D, Liberati A, Tetzlaff J, et al. Preferred reporting items for systematic reviews and meta-analyses: the PRISMA statement. PLoS Med 2009;6:e1000097.
12 Critical appraisal skills programme. CASP checklists, 2020. Available: https://casp-uk.net/casp-tools-checklists/

13 Thomas J, Harden A. Methods for the thematic synthesis of qualitative research in systematic reviews. BMC Med Res Methodol 2008;8:45.

14 The GRADE Working Group. Welcome to the grade Working group 2004-2020, 2020. Available: https://www.gradeworkinggroup.org/

15 The GRADE CERQual project group. GRADE CERQual, 2018. Available: https://www.cerqual.org/

16 Bray N, Kolehmainen N, McAnuff J. Early mobility and powered wheelchair evidence review (empower): examining the effectiveness and cost-effectiveness of earlier provision of powered mobility interventions for children with mobility limitations, 2020.

17 NHS Digital. NHS health check programme, patients recorded as attending and not attending, 2012-13 to 2017-18, 2021. Available: https://digital.nhs.uk/data-and-information/publications/statistical/ nhs-health-check-programme/2012-13-to-2017-18.

18 Chang KC-M, Lee JT, Vamos EP, et al. Impact of the National health service health check on cardiovascular disease risk: a difference-indifferences matching analysis. CMAJ 2016;188:E228-38.

19 Coghill N, Garside L, Montgomery AA, et al. Nhs health checks: a cross- sectional observational study on equity of uptake and outcomes. BMC Health Serv Res 2018;18:238.

20 Chattopadhyay K, Biswas M, Moore R. Nhs health check and healthy lifestyle in Leicester, England: analysis of a survey dataset. Perspect Public Health 2020;140:27-37.

21 Lang S-J, Abel GA, Mant J, et al. Impact of socioeconomic deprivation on screening for cardiovascular disease risk in a primary prevention population: a cross-sectional study. BMJ Open 2016;6:e009984.

22 Woringer M, Cecil E, Watt $\mathrm{H}$, et al. Evaluation of community provision of a preventive cardiovascular programme - the National Health Service Health Check in reaching the under-served groups by primary care in England: cross sectional observational study. BMC Health Serv Res 2017;17:405

23 Public Health England. NHS health check, 2020. Available: https:// fingertips.phe.org.uk/profile/nhs-health-check-detailed/data\#page/0/ page-options/ovw-do-0 [Accessed 24 Nov 2020].

24 McDermott L, Cornelius V, Wright AJ, et al. Enhanced invitations using the Question-Behavior effect and financial incentives to promote health check uptake in primary care. Ann Behav Med 2018;52:594-605.

25 Attwood S, Morton K, Sutton S. Exploring equity in uptake of the NHS health check and a nested physical activity intervention trial. $J$ Public Health 2016;38:560-8.

26 Coghill N, Garside L. A quantitative quasi-experimental approach to the evaluation of a telephone outreach service. University of Bath, 2016.

27 Dalton ARH, Bottle A, Okoro C, et al. Uptake of the NHS health checks programme in a deprived, culturally diverse setting: crosssectional study. J Public Health 2011;33:422-9.

28 Cornelius VR, McDermott L, Forster AS, et al. Automated recruitment and randomisation for an efficient randomised controlled trial in primary care. Trials 2018;19:341.

29 Gulliford MC, Khoshaba B, McDermott L. Cardiovascular risk at health checks performed opportunistically or following an invitation letter. J Public Health 2017:1-6.

30 Gidlow CJ, Ellis NJ, Riley V, et al. Randomised controlled trial comparing uptake of NHS health check in response to standard letters, risk-personalised letters and telephone invitations. BMC Public Health 2019;19:224.

31 Gold N, Durlik C, Sanders JG, et al. Applying behavioural science to increase uptake of the NHS health check: a randomised controlled trial of gain- and loss-framed messaging in the National patient information leaflet. BMC Public Health 2019;19:14. doi:10.1186/ s12889-019-7754-5

32 Sallis A, Sherlock J, Bonus A, et al. Pre-notification and reminder SMS text messages with behaviourally informed invitation letters to improve uptake of NHS health checks: a factorial randomised controlled trial. BMC Public Health 2019;19:1162.

33 Sallis A, Bunten A, Bonus A, et al. The effectiveness of an enhanced invitation letter on uptake of national health service health checks in primary care: a pragmatic quasi-randomised controlled trial. BMC Fam Pract 2016;17:8. doi:10.1186/s12875-016-0426-y

34 Harrop N, Alpsten T. Saving lives through effective patient engagement around NHS health checks. Clin Gov 2015. doi:10.1108/ CGIJ-08-2015-0025

35 Stone TJ, Brangan E, Chappell A, et al. Telephone outreach by community workers to improve uptake of NHS health checks in more deprived localities and minority ethnic groups: a qualitative investigation of implementation. J Public Health 2020;42:e198-206. doi:10.1093/pubmed/fdz063 
36 Roberts DJ, de Souza VC. A venue-based analysis of the reach of a targeted outreach service to deliver opportunistic community NHS Health Checks to 'hard-to-reach' groups. Public Health 2016:137:176-81.

37 Whittaker PJ. Uptake of cardiovascular health checks in community pharmacy versus general practice. J Fluid Mech 2019;884:6. doi:10.1002/jppr.1568

38 Alageel S, Gulliford MC. Health checks and cardiovascular risk factor values over six years' follow-up: matched cohort study using electronic health records in England. PLoS Med 2019;16:16.

39 Alageel S, Gulliford MC, McDermott L, et al. Implementing multiple health behaviour change interventions for cardiovascular risk reduction in primary care: a qualitative study. BMC Fam Pract 2018;19:171.

40 Alageel S, Gulliford MC, Wright A, et al. Engagement with advice to reduce cardiovascular risk following a health check programme: a qualitative study. Health Expect 2020;23:193-201. doi:10.1111/ hex.12991

41 Hawking MKD, Timmis A, Wilkins F, et al. Improving cardiovascular disease risk communication in NHS health checks: a qualitative study. BMJ Open 2019;9:e026058.

42 Palladino R, Vamos E, Chang KCM, et al. Impact of a national diabetes risk assessment and screening programme in England: a quasi-experimental study. The Lancet 2017;390:S65. doi:10.1016/ S0140-6736(17)33000-3

43 Kennedy O, Su F, Pears R, et al. Evaluating the effectiveness of the $\mathrm{NHS}$ health check programme in South England: a quasi-randomised controlled trial. BMJ Open 2019;9:e029420.

44 Robson J, Dostal I, Madurasinghe V, et al. Nhs health check comorbidity and management: an observational matched study in primary care. Br J Gen Pract 2017;67:e86-93.

45 Alageel S, Wright A, Gulliford M. Impact of the health check programme on the provision of smoking cessation interventions in England. Eur J Public Health 2017;27. doi:10.1093/eurpub/ ckx187.184

46 Collins B, Kypridemos C, Cookson R. Universal or targeted cardiovascular screening? modelling study using a sector-specific distributional cost effectiveness analysis. Prev Med 2020;105879. doi:10.1016/j.ypmed.2019.105879

47 Collins B, Kypridemos C, Parvulescu P. The cost-effectiveness and equity of the NHS health checks cardiovascular disease prevention programme: a microsimulation using real-world data from a deprived Northern City 2017;71. doi:10.1136/jech-2017-SSMAbstracts.99

48 Hinde S, Bojke L, Richardson G, et al. The cost-effectiveness of population health checks: have the NHS health checks been unfairly maligned? Z Gesundh Wiss 2017;25:1-7.

49 Robson J, Garriga C, Coupland C, et al. NHS health checks: an observational study of equity and outcomes 2009-2017. Br J Gen Pract 2021;71:e701-10. doi:10.3399/BJGP.2020.1021

50 Garriga C, Robson J, Coupland C, et al. Nhs health checks for people with mental ill-health 2013-2017: an observational study. Epidemiol Psychiatr Sci 2020;29:e188.

51 Gold N, Tan K, Sherlock J, et al. Increasing uptake of NHS health checks: a randomised controlled trial using GP computer prompts. Br J Gen Pract 2021;71:e693-700. doi:10.3399/BJGP.2020.0887

52 Williams M, Thomson L, Butcher E, et al. Nhs health check programme: a qualitative study of prison experience. $J$ Public Health 2020. doi:10.1093/pubmed/fdaa189. [Epub ahead of print: 20 Nov 2020].

53 Paxton B, Mills K, Usher-Smith JA. Fidelity of the delivery of NHS health checks in general practice: an observational study. BJGP Open 2020;4. doi:10.3399/bjgpopen20X101077. [Epub ahead of print: 27 Oct 2020].

54 Gidlow CJ, Ellis NJ, Riley V, et al. Cardiovascular disease risk communication in NHS health checks: a qualitative videostimulated recall interview study with practitioners. BJGP Open 2021;5. doi:10.3399/BJGPO.2021.0049. [Epub ahead of print: 26 Oct 2021].

55 Riley V, Ellis NJ, Cowap L, et al. A qualitative exploration of two risk calculators using video-recorded NHS health check consultations. BMC Fam Pract 2020;21:1-13.

56 Alageel S, Gulliford MC, Wright A, et al. Engagement with advice to reduce cardiovascular risk following a health check programme: a qualitative study. Health Expect 2020;23:193-201.

57 Palladino R, Vamos EP, Chang KC-M, et al. Evaluation of the diabetes screening component of a national cardiovascular risk assessment programme in England: a retrospective cohort study. Sci Rep 2020;10:1-11.

58 Burns J, Polus S, Brereton L, et al. Looking beyond the forest: using harvest plots, gap analysis, and expert consultations to assess effectiveness, engage stakeholders, and inform policy. Res Synth Methods 2018;9:132-40. 